\section{Health-Info-Net: Die Chance jetzt nutzen!}

\author{
F.-X. Deschenaux ${ }^{1}$, G. von Below ${ }^{2}$ \\ 1 VR-Präsident \\ ${ }^{2}$ Geschäftsführer
}

Mit dem Health-Info-Net (HIN) hat die FMH vor drei Jahren den Aufbau einer technisch hochstehenden Internetplattform lanciert, die international anerkanntem Sicherheitsstandard entspricht. Sie bietet bereits über 2000 Ärztinnen und Ärzten eine Vielzahl von attraktiven Möglichkeiten zur Nutzung des Internets bei ihrer täglichen Arbeit.

\section{Welche Überlegungen führten zum Aufbau von HIN?}

Die wesentlichen Gründe für den Aufbau einer speziell auf die Bedürfnisse der Medizinalpersonen ausgerichteten Plattform durch die FMH waren folgende:

\section{Zugang zu Wissen und Fakten}

Bei HIN finden Ärztinnen und Ärzte rasch und unkompliziert Daten und Fakten zu wissenschaftlichen Arbeiten und Projekten sowie Informationen über Spitäler, Firmen und andere Gruppierungen aus dem Gesundheitswesen. HIN ist eine Plattform, über welche die Ärzteschaft und ihre Partner im Gesundheitswesen Informationen austauschen und Geschäftsbeziehungen unterhalten können.

\section{Sicherheit im Internet}

HIN schützt Ihre Daten vor unbefugtem Zugriff! Die eingesetzten Zugangskontrollen, Benutzerauthentifizierungen und Verschlüsselungstechnologien erlauben Ihnen den gesicherten Austausch sensibler Daten mit anderen Teilnehmern: sei es per E-mail oder in geschützten Diskussionsforen. Wer sich via HINPower-Providing einwählt, hat zudem seinen Computer sicher gegen Hacker und andere Attacken aus dem Internet geschützt.

\section{Information und Support}

Wer ein HIN-Abonnement gelöst hat, kann bei Bedarf via Supportline auf die kompetente Hilfe und Unterstützung eines erfahrenen und qualifizierten Supportteams zurückgreifen, das den Benutzern mit Rat und Tat zur Seite steht. Tips und Service sind wertvoll, die Preise vernünftig.

\section{Diffusion des Internets nimmt rasant zu!}

Branchenexperten rechnen damit, dass bis Ende dieses Jahres jeder dritte, wenn nicht gar jeder zweite Haushalt über einen Internetanschluss verfügen wird. Heute haben in der Schweiz rund 35\% der Ärztinnen und Ärzte einen Internetanschluss. Davon sind rund 2000 HIN-Abonnenten. Sie erhalten für die jährlichen Abonnementsgebühren von heute Fr. 180.- eine ganze Reihe von Dienstleistungen und Zugriffsrechten sowie eine stabile, technisch hervorragend geschützte Plattform für den Austausch ihrer sensiblen, da patientenbezogen, Daten im Internet.

Dennoch sind heute bei vielen FMH-Mitgliedern noch Zweifel an den Vorteilen des Internets und des HIN vorhanden und sie haben aus diesen oder anderen Gründen sich noch nicht für einen Anschluss entschieden:

- Für manche Ärztinnen und Ärzte ist nicht zwingend ersichtlich, weshalb der Schritt ins elektronische Zeitalter notwendig sein sollte.

- Man hegt Zweifel an der Kosten-/Nutzen-Relation - und schliesslich gibt es ja genügend Anbieter, die mit attraktivem - allerdings dann eben nicht geschütztem! - Gratiszugang zum Internet werben.

- Ein aktiver Druck von aussen bestand bisher nicht; die moderne Technologie kann zudem abschreckend wirken: Mit dem guten alten Papier ist es bisher schliesslich auch gegangen.

- Verständliche Ängste sind auch bezüglich Datenschutz und Datenmissbrauch vorhanden: Wer, welche Institutionen könnte allenfalls unbefugt Einsicht nehmen in die Daten der Praxis, des Spitals oder gar der ganzen Ärzteschaft, wenn sich die Leistungserbringer über eine gemeinsame Plattform austauschen? Pressemeldungen über Viren wie Melissa oder ILOVEYOU, die weltweit Milliardenschäden verursacht haben sollen, tragen nicht dazu bei, Skepsis und Ängste bezüglich Internet abzubauen. Nur: Hier geht es nicht um HIN-spezifische Probleme - sie betreffen sämtliche Provider in gleichem Masse. Was den Datenschutz betrifft, sind im Gegenteil zu HIN gerade und erst recht berechtigte Bedenken bei einem normalen Internetanschluss und beim E-mailVerkehr über alle kommerziellen Provider angebracht.

\section{Warum ein HIN-Abonnement für alle FMH- Mitglieder?}

Das HIN strebt - als Gesellschaft, welche mehrheitlich im Besitz der FMH ist - nach der bisherigen Aufbauphase einen Systemwechsel bezüglich Finanzierung an: weg von der Plattform, die grösstenteils durch Einzelabonnemente finanziert wird, hin $\mathrm{zu}$ einer selbsttragenden Struktur, die sich über die Dienstleistungsangebote kommerzieller Anbieter selber finanziert. HIN bietet im rasch wachsenden Markt der kommerziellen Nutzung des Internets bereits
Korrespondenz:

Georg von Below

Health-Info-Net AG

Zürichstrasse 64

CH-8700 Küsnacht 
heute jedem Anbieter, welcher mit niedergelassenen Ärzten kommunizieren will, eine ideale Infrastruktur an. Ob Spitäler, Anbieter von Labordiensten, Lieferanten von Medikamenten- oder Verbrauchsmaterialien: sie alle schätzen die Vorteile einer gesicherten Plattform, auf welcher die Geschäftspartner identifiziert und ihre Meldungen mit einer elektronischen Signatur versehen sind. Denn nur so lassen sich vertrauenswürdige Geschäftsbeziehungen über das Internet abwickeln.

HIN als Plattform ist für die unterschiedlichen Anbieter aber nur dann interessant, wenn zwei Bedingungen erfüllt sind:

1. Der technologische Standard muss den besonderen Anforderungen des Datenschutzes im Gesundheitswesen standhalten können.

$\Rightarrow$ Die von HIN eingesetzte ASAS-Technologie erfüllt diese Kriterien und wird auch von Experten als State-of-the-Art-Technologie gelobt. Bereits setzen auch erste Banken und Spitäler die ASASTechnologie ein.

2. Möglichst rasch müssen möglichst viele - am besten alle! - FMH-Mitglieder über die HIN-Plattform ansprechbar sein. Ähnlich, wie die Schweizerische Ärztezeitung auch alle FMH-Mitglieder erreicht, soll auch das HIN als Informationskanal zur Ärzteschaft genutzt werden können.

Ohne diese beiden Prämissen ist HIN für potentielle finanzkräftige Anbieter nicht genügend interessant und - was den künftigen Informationsaustausch sehr viel komplizierter und unpraktischer gestalten wird: zahlreiche Anbieter werden ihre eigenen Sicherheitssysteme aufbauen.

$\Rightarrow$ HIN bietet in dieser Beziehung eine einheitliche, technologisch absolut elegante und polyvalent nutzbare Technologie, welche alle Prämissen erfüllt, um einen technologischen Sicherheitsstandard im Gesundheitswesen etablieren zu können.

\section{Antrag HIN-Abonnement für alle FMH-Mitglieder}

Um dieses Ziel zu erreichen, hat der Zentralvorstand der FMH entschieden, der Ärztekammer vom 21./22. Juni 2000 folgenden Antrag zu unterbreiten:

1. Zur Förderung der Nutzung des Internets durch die Ärztinnen und Ärzte in der Schweiz soll jedes Mitglied der FMH das heutige HIN-Abonnement kostenlos erhalten.

2. Die Finanzierung dieser Absicht soll durch eine auf drei Jahre beschränkte Erhebung eines zweckgebundenen Sonderbeitrags von Fr. 50.- pro Jahr und FMH-Mitglied sichergestellt werden. Während dieser Zeitspanne soll sich - wie vorne erwähnt das HIN zu einer selbsttragenden Struktur entwickeln, die sich über kommerzielle Anbieter selber finanziert.

\section{Welche Vorteile sind damit für Sie verbunden?}

Allen, die heute schon ein HIN-Abonnement besitzen, bringt diese Aktion folgende Vorteile:

- Die Kosten für das jährliche HIN-Abonnement werden massiv reduziert und können mit der Zahlung des FMH-Beitrages erledigt werden.

- Durch die angestrebte Promotion werden neue Kollegen ermutigt, in die Internettechnologie einzusteigen. Die Anzahl der Kommunikationspartner, mit welchen auch sensitive Informationen ausgetauscht werden können, wird stetig steigen.

- Die Plattform HIN wird als "Marktplatz" für die übrigen Partner im Gesundheitswesen sowie für die Anbieter von Dienstleistungen interessant: Hier können sie den HIN-Abonnenten neue Produkte und Geschäftsmöglichkeiten anbieten.

- Für die heute noch nicht mit Internet und HIN vertrauten Ärztinnen und Ärzte bietet die Aktion Gelegenheit und Anreiz, sich mit dieser neuen Technologie vertraut zu machen.

- Die Kosten für das jährliche HIN-Abonnement sind neu viel tiefer als bisher und bereits mit dem FMH-Beitrag bezahlt. Schade, wenn diese Gelegenheit nicht genutzt wird!

- Der Einstieg ins Internetzeitalter wird mit speziellen Aktionen für den Kauf von "Plug \& Surf»Laptops (nur einstecken - und schon bin ich im Internet!) mit bereits fertig installiertem Anschluss ans Internet und weiteren Internet-Einsteigerkursen zusätzlich unterstützt.

- Sie können Schritt für Schritt in die vielfältigen Möglichkeiten des Internets hineinwachsen, bevor Sie es für kommerzielle Anwendungen einsetzen.

\section{Die Chance jetzt nutzen!}

Das HIN hat sich zu einer Plattform entwickelt, welche sich im nationalen und internationalen Vergleich sehen lassen kann. Die e-Kommunikation und das e-Business werden auch vor dem Gesundheitswesen nicht halt machen. Jetzt ist der Moment gekommen, dass sich die Ärztinnen und Ärzte für einen gemeinsamen Standard entscheiden, bevor ihnen das Heft aus der Hand genommen wird.

Der ZV hofft an der Ärztekammersitzung vom 21./22. Juni 2000 auf Ihre Unterstützung. Helfen Sie mit, diese Chance jetzt zu nutzen! 